\title{
Broadband Spectroscopy of Nanoporous-Gold Promoter
}

\author{
S. Kuwano-Nakatani ${ }^{1}$, Y. H. Han ${ }^{1}$, T. Takahashi ${ }^{2}$ and T. Awano ${ }^{1 *}$ \\ ${ }^{1}$ Tohoku Gakuin University, Japan \\ ${ }^{2}$ Research Reactor Institute, Kyoto University, Japan \\ *corresponding author: awano@tjcc.tohoku-gakuin.ac.jp
}

\begin{abstract}
The efficiency of UV photocatalysis on $\mathrm{TiO}_{2}$ particles was increased by mixing $\mathrm{TiO}_{2}$ particles with nanoporous gold (NPG) with pore diameters of $10-40 \mathrm{~nm}$. This means that NPG acts as a promoter in the photocatalytic reaction of $\mathrm{TiO}_{2}$. Broadband spectroscopic results from millimeter wave to ultra violet of NPG membrane are discussed to estimate plasmonic effect on the catalysis.
\end{abstract}

\section{Introduction}

It is well known that bulk $\mathrm{Au}$ is inert. However, $\mathrm{Au}$ nanoparticles with several-nanometer-scale sizes are catalytically active for several chemical reactions such as the decomposition of formaldehyde and oxidation of $\mathrm{CO}^{1,2}$. The catalytic activity depends on the size of nanoparticles. The nanoparticles with diameters in the range of 2 to $5 \mathrm{~nm}$ show the highest activity for oxidation ${ }^{3}$. Nanoporous $\mathrm{Au}$ (NPG) with $10-50 \mathrm{~nm}$ pore size also shows catalytic activity for oxidation $^{2}$.

Recently, $\mathrm{TiO}_{2}$ is being widely used for photocatalysis of various materials. $\mathrm{TiO}_{2}$ has a different activity with crystal structure for different wavelengths. It has been reported that composite materials of $\mathrm{TiO}_{2}$ with $\mathrm{Au}$ or $\mathrm{Pt}$ nanoparticles enhance $\mathrm{TiO}_{2}$ catalytic activity ${ }^{4} \mathrm{TiO}_{2}$ plays an active role in the decomposition of water to generate $\mathrm{OH}$ radicals. It is supposed that the plasmonic electromagnetic effect of $\mathrm{Au}$ or Pt enhances the catalytic activity of $\mathrm{TiO}_{2}$. In addition, since pores of NPG are able to trap the $\mathrm{TiO}_{2}$ particles on its surface, NPG seems to be a photocatalytic nanocomposite because of enhancement of the catalytic activity by the electromagnetic effect. We have found that NPG acts as a promoter to produce $\mathrm{OH}$ radicals using $\mathrm{TiO}_{2}{ }^{5}$. We report optical properties of NPG in this paper to investigate electromagnetic and plasmonic origin of the promotor effect.

\section{Experimental}

NPG films were obtained by nitric acid etching of Ag from $\mathrm{Au}_{35} \mathrm{Ag}_{65}$ alloy films with about $100 \mathrm{~nm}$ thickness. The average pore diameter was $10-40 \mathrm{~nm}$, according to the dealloying temperature and time. Figure 1 shows SEM images of NPG films with average pore diameters of $20 \mathrm{~nm}$ (a) and $42 \mathrm{~nm}$ (b). The other samples were confirmed to have average pore diameters of 11 and $33 \mathrm{~nm}$.

Reflectivity spectra of the NPG films on quartz plate were measured over four regions: (1) millimeter wave region was measured using a coherent transition radiation induced by the LINAC facility of Research Reactor Institute, Kyoto University; (2) far-infrared region was measured using synchrotron radiation by UVSOR facility of Institute for Molecular Science. This region was also measured using a Martin-Puplett interferometer JASCO FARIS-1 with a
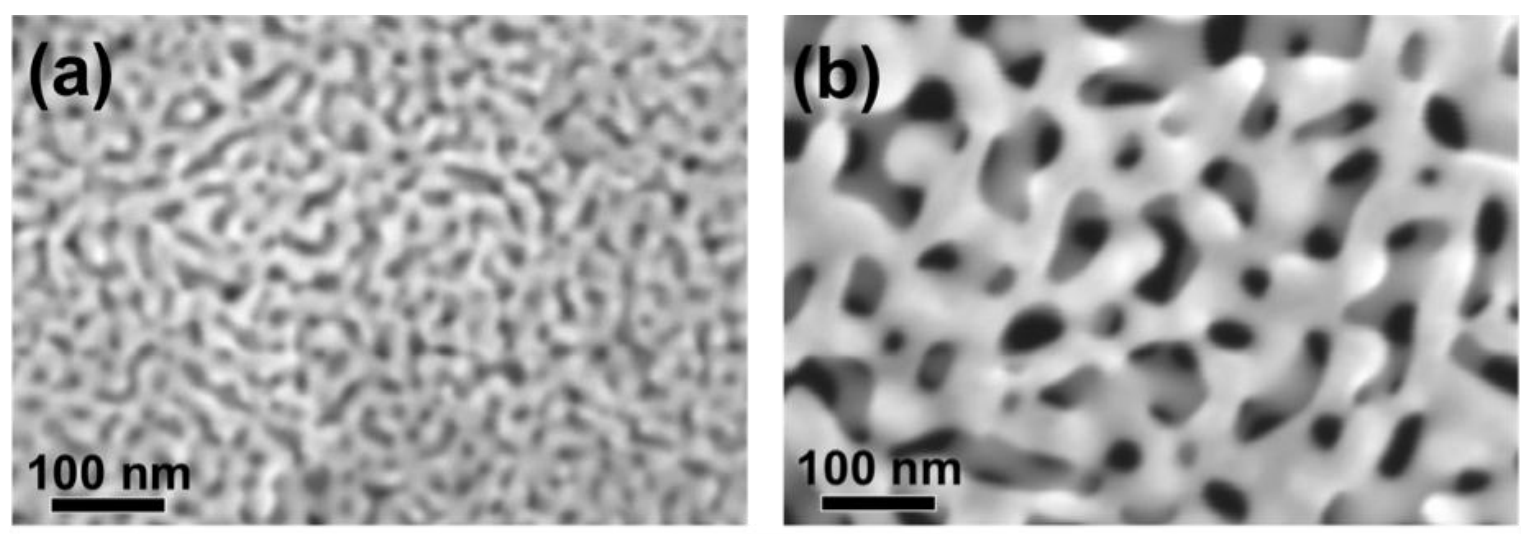

Figure 1: SEM images of NPG films. Pore diameter is (a) $20 \mathrm{~nm}$ and (b) $42 \mathrm{~nm}$. 
conventional mercury light source; (3) infrared region was measured using JASCO FT-4100 spectrometer; (4) near infrared, visible and ultraviolet region.

\section{Results and discussion}

\subsection{Promoter effect}

Anatase (25 $\mathrm{nm}$ average diameter) and rutile ( $85 \mathrm{~nm}$ average diameter) $\mathrm{TiO}_{2}$ particles were in the ratio $4: 1$. The mixture of

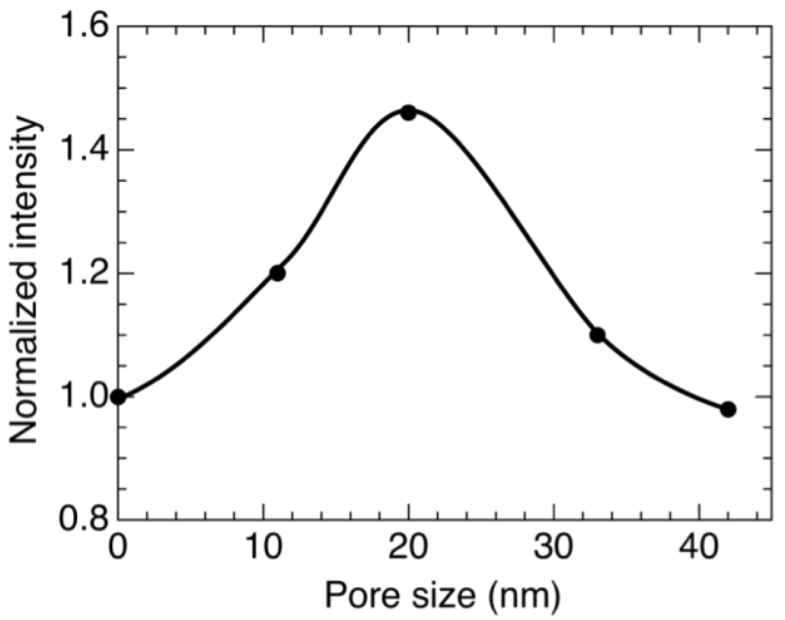

Figure 2: OH-radical production efficiency as a function of average pore diameter.
NPG, $\mathrm{TiO}_{2}$, and spin trapping agent was irradiated by Blacklight with wave length $365 \mathrm{~nm}$. The spectra for the estimation of the amount of induced $\mathrm{OH}$ radicals were measured by $\mathrm{X}$-band ESR. No $\mathrm{OH}$ radical was formed by the $\mathrm{Au}_{35} \mathrm{Ag}_{65}$ alloy film or NPG film alone during the Blacklight irradiation. Besides, the coexistence of $\mathrm{TiO}_{2}$ particles and NPG films enhanced the production of the $\mathrm{OH}$ radical. ESR signals of the $\mathrm{OH}$-radical spin adduct were observed, and it was confirmed that NPG is a promoter for photocatalysis on $\mathrm{TiO}_{2}$. Fig. 2 shows the efficiency of $\mathrm{OH}$ radical productivity vs. average pore sizes. The maximum efficiencies normalized by that of an $\mathrm{Au}_{35} \mathrm{Ag}_{65}$ alloy film was 1.5 at average pore diameter of $20 \mathrm{~nm}$. No enhancement was observed at pore diameters over $40 \mathrm{~nm}$. This peak seems to be due to that the average particle diameter of the anatase $\mathrm{TiO}_{2}$ particles, which are sensitive to the irradiated Black-light, was $25 \mathrm{~nm}$. Therefore, the active anatase $\mathrm{TiO}_{2}$ particles appear to dispersively infiltrate into the pores of $\mathrm{Au}$ and tightly stick to the pore surface. This seems to enhance the promoter effect of $\mathrm{Au}$ on $\mathrm{TiO}_{2}$.

\subsection{Spectroscopic results}

\subsubsection{Reflectivity spectra}

Fig. 3 shows broadband reflectivity spectra of NPG with various average pore sizes. The horizontal axis is in a logarithm to show the wide spectral range. Fine structures in IR spectra are due to $\mathrm{CO}_{2}$ and $\mathrm{H}_{2} \mathrm{O}$ in atmosphere. Measurement of the lack region at terahertz wave is under way at the UVSOR facility. Peaks at $0.06 \mathrm{eV}$ are due to reflection of the substrate quartz. It is especially strong at

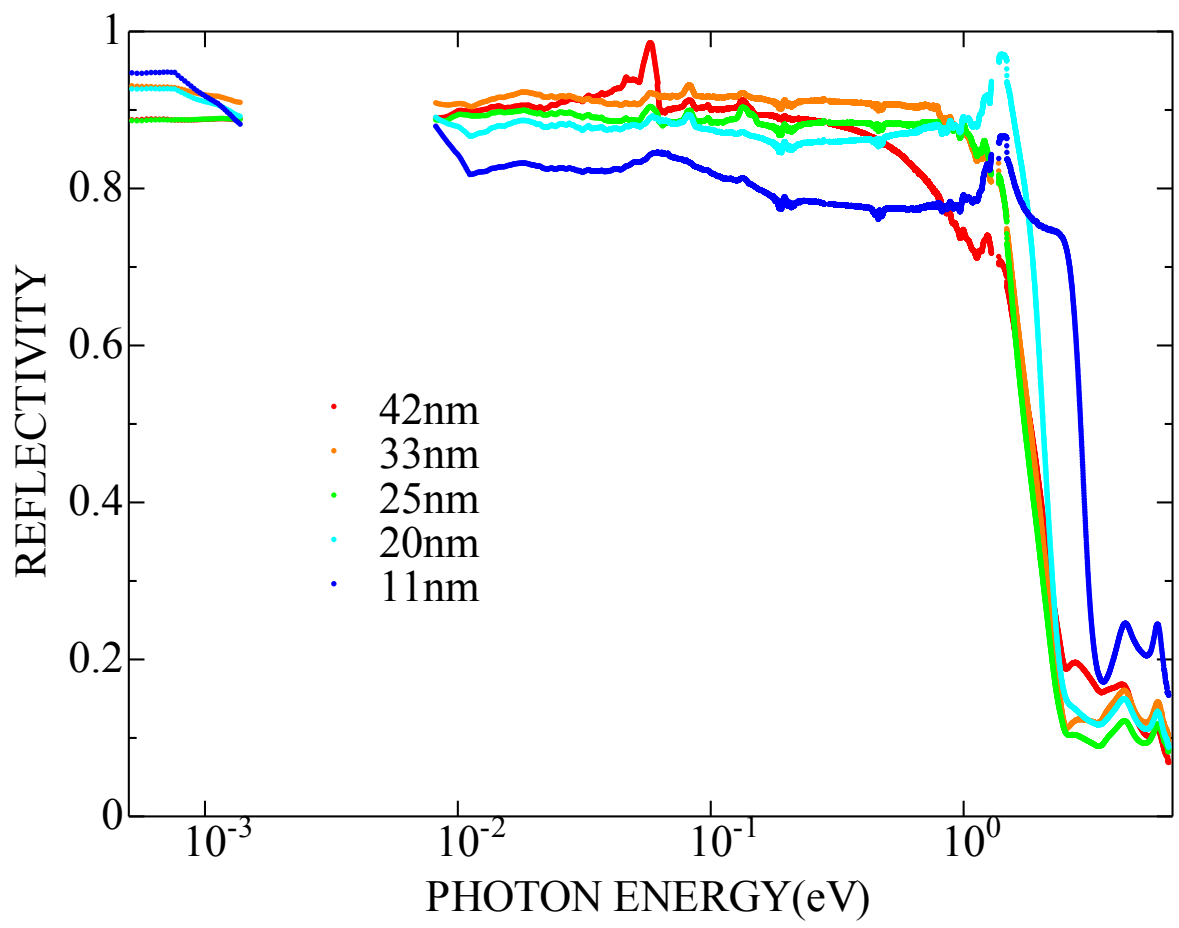

Figure 3: Reflectivity spectra of NPG membranes on quartz substrates. Average pore diameters of the five samples with diameter on the order of $42 \mathrm{~nm}$ to $11 \mathrm{~nm}$ are shown in the NIR-UV figure. 


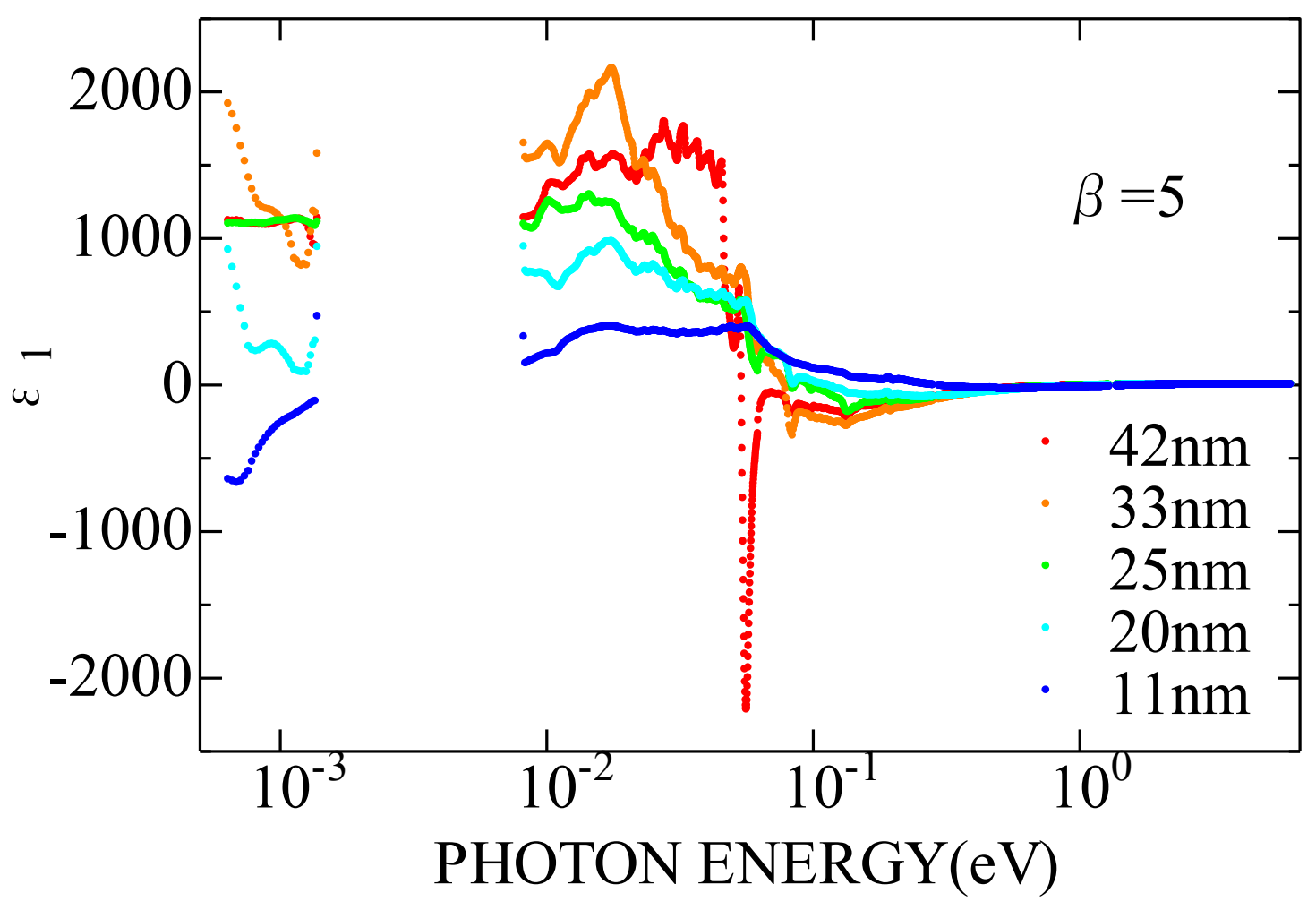

Figure 4: Dielectric constant spectra (real part) of NPG membranes on quartz substrates. The extrapolation exponent index of the reflectivity at high energy $\beta=5$.

$42 \mathrm{~nm}$ pore sample because of void content (low average density) of the large porous thin film. Small structures at infrared region are due to absorption by $\mathrm{CO}_{2}$ and $\mathrm{H}_{2} \mathrm{O}$ in atmosphere. Peaks at $1.45 \mathrm{eV}$ are due to reflectivity dip of the reference Al mirror. Plasmonic characteristic spectral changes were observed from IR to UV region. They depend on the pore size.

\subsubsection{Dielectric constants}

Dielectric constants are obtained by Kramer-Kronig analysis. The measured reflectivity spectra were wide enough to obtain dielectric constants by calculation from it. The reflectivity spectra were extrapolated as constant to connect smoothly at low energy side and terahertz region. They were extrapolated as exponential decrease function to connect smoothly. The optimum exponent coefficients were scattered largely. Fig. 4 and 5 show dielectric constants at the exponent $\beta=5$ to see the pore size dependence of optical properties. Fig. 4 shows real parts of the dielectric constant. The large structures at $0.06 \mathrm{eV}$ are due to the reflection of the substrate quartz. Spectra at low energy region change as sequent by pore sizes except the case of $42 \mathrm{~nm}$. This seems to be due to low average density of gold by pore volume.

Fig. 5 shows imaginary parts of the dielectric constant. Lower figure shows a log-log plot to show the region from infrared to UV. The large structures at $0.06 \mathrm{eV}$ are due to the reflection of the substrate quartz. Spectra at low energy region change as sequent by pore sizes except the case of $42 \mathrm{~nm}$ as in the $\varepsilon_{1}$ spectra. It is clearly observed in the lower figure that the spectra of large and intermediate pore sizes show linear decrease which means these spectra decrease as power function in the region from 0.1 to $1 \mathrm{eV}$. The $\varepsilon_{2}$ spectra shows absorption intensity for transverse wave. To investigate plasmonic absorption for longitudinal wave, energy loss function spectra obtained from these dielectric constants spectra are discussed in the next section. 

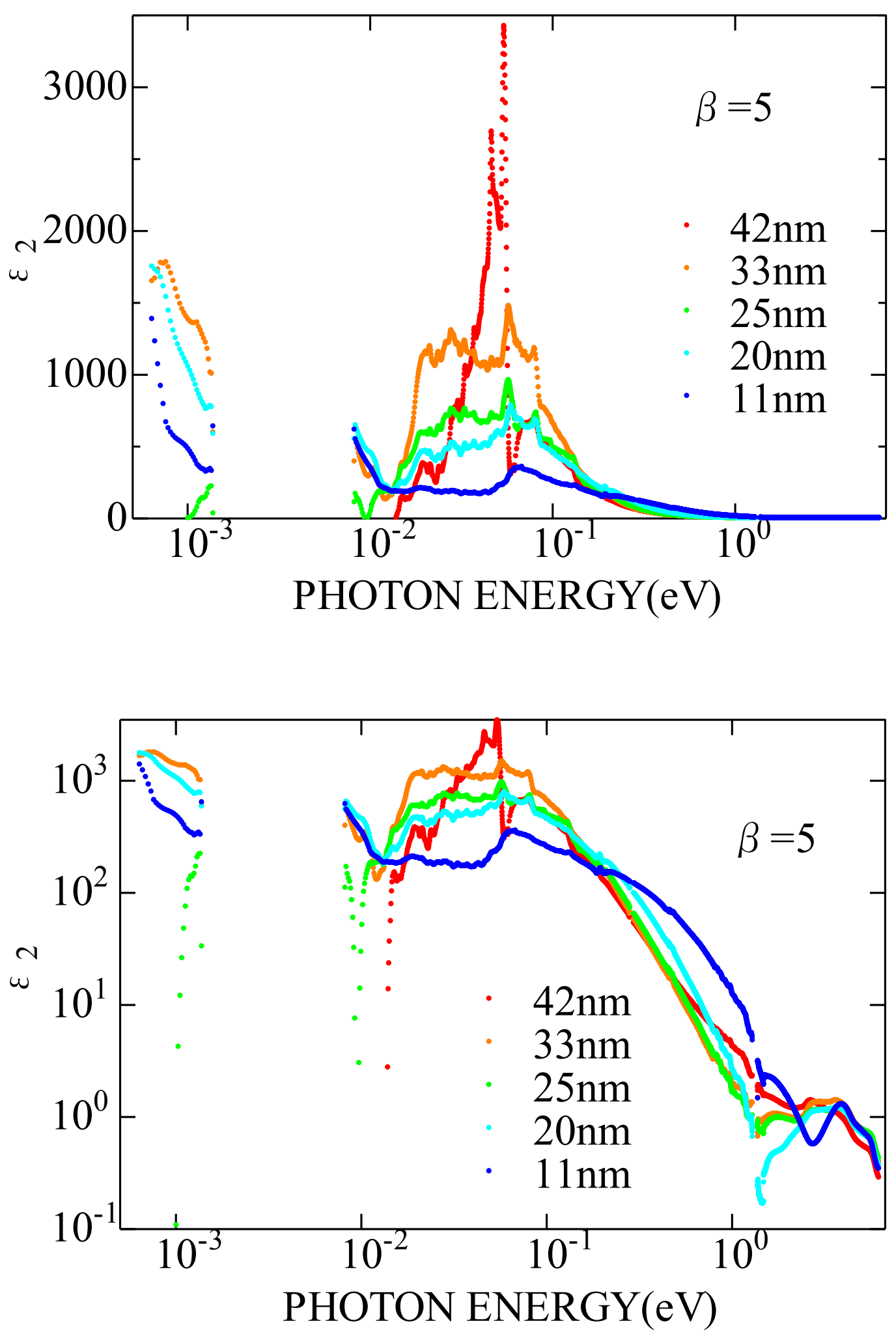

Figure 5: Dielectric constant spectra (imaginary part) of NPG membranes on quartz substrates. The above figure is semi-log plot and the below one is log-log plot. The extrapolation exponent index of the reflectivity at high energy $\beta=5$. 


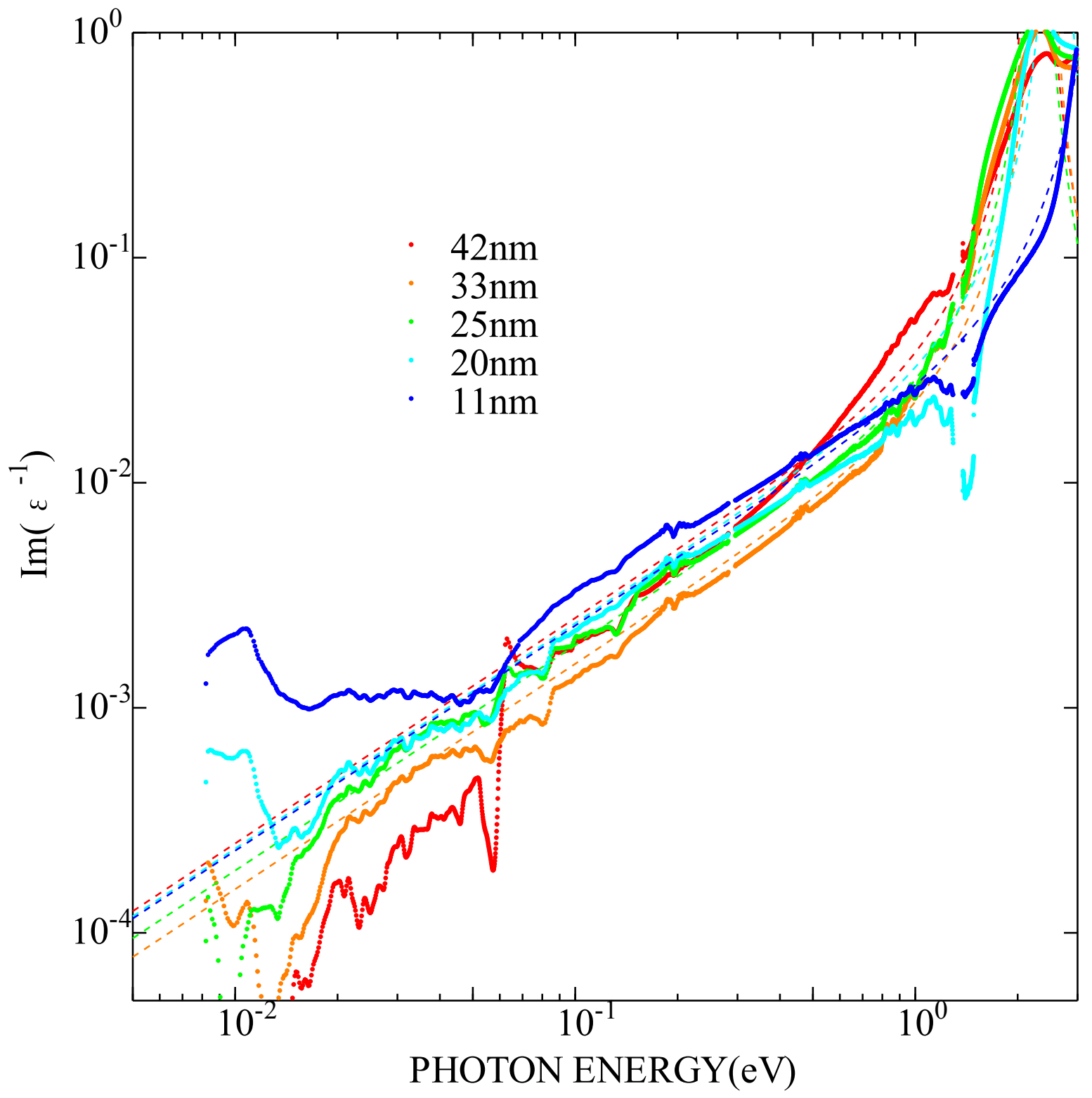

Figure 6: Drude curve fitting of the energy loss function spectra of NPG membranes on quartz substrates. Dashed lines show fitting curves for each pore size sample.

\subsubsection{Plasmon fitting}

Plasmon fitting by classical Drude model in energy loss function spectra is shown in fig. 6. In Drude model, dielectric constant is

$\varepsilon=\varepsilon_{\infty}\left(1-\frac{\omega_{p}^{2}}{\omega^{2}+\gamma^{2}}+i \frac{\gamma \omega_{p}{ }^{2}}{\omega\left(\omega^{2}+\gamma^{2}\right)}\right)$,

where $\omega_{p}=\sqrt{\frac{4 \pi N e^{2}}{m^{*} \varepsilon_{\infty}}}$ is the plasma frequency, $\gamma$ is

dumping factor and $\varepsilon_{\infty}$ is dielectric constant at high frequency. Energy loss function, $\operatorname{Im}(1 / \varepsilon)$, is proportional to absorption intensity by transverse wave. The plasmon fitting parameters are summarized in Table 1. Systematic decrease of the plasma frequency with pore size increment was observed. Plasma frequency of bulk gold is $8.6 \mathrm{eV}$, therefore, these $\omega_{\mathrm{p}}$ for pore samples are much small. The promoter effect seems to be related with small values of these parameters. The origin of pore size dependence of the promoter effect is still not clear.

Table 1: Plasmon parameters.

\begin{tabular}{cccc}
\hline pore size $(\mathrm{nm})$ & $\omega_{\mathrm{p}}(\mathrm{eV})$ & $\gamma(\mathrm{eV})$ & $\varepsilon_{\infty}$ \\
\hline 42 & 2.3 & 0.06 & 2.2 \\
33 & 2.4 & 0.01 & 9 \\
25 & 2.3 & 0.025 & 4 \\
20 & 2.6 & 0.02 & 8 \\
11 & 3.6 & 0.03 & 10 \\
\hline
\end{tabular}




\section{Conclusions}

Promoter effect was discovered on NPG/TiO 2 system. The detail enhance mechanism of the promoter effect is not clear yet. However, we could observe that smaller plasmon parameters on NPG than those on bulk gold.

\section{Acknowledgements}

Part of this work was done under the visiting researcher program of Research Reactor Institute, Kyoto University and UVSOR facility of Institute for Molecular Science.

\section{References}

[1] Bierner, J. et al., Surface-chemistry-driven actuation in nanoporous gold, Nature Materials 8: 47-51, 2009.

[2] Wittstock, A., V. Zielasek, J. Biener, C. M. Friend, M. Bäumer, Nanoporous Gold Catalysis for Selective GasPhase Oxidative Coupling of Methanol at Low Temperature, Science 327: 319-322, 2003.

[3] Haruta, M., When Gold Is Not Noble: Catalysis by Nanoparticles, Chem. Rec..3: 75-87, 2003.

[4] Haruta, M., Nanoparticulate Gold Catalysis for LowTemperature CO Oxidation, J. New Mat. Electrochem. Sys. 7: 163-172, 2004.

[5] Kuwano-Nakatani S., T. Ando, K. Oba and Y.H. Han, to be published. 